(REVIEW ARTICLE)

\title{
Is thrombocytopenia considered a valuable indicator tool for malaria?
}

\author{
Asaad Ma. Babker* \\ Department of Medical Laboratory Sciences, College of Health Sciences, Gulf Medical University, Ajman, UAE.
}

Publication history: Received on 09 March 2020; revised on 19 March 2020; accepted on 21 March 2020

Article DOI: https://doi.org/10.30574/gscarr.2020.2.3.0019

\begin{abstract}
Malaria is a major public health problem especially in tropical and sub-tropical regions around the world with varied hematological consequences. Thrombocytopenia is common in the laboratory finding among patients with malaria. Mechanism of thrombocytopenia in malaria is till now not clearly known. In this review we try to evaluate the presence of thrombocytopenia during malaria as valuable Indicator tool for presence of Malaria.
\end{abstract}

Keywords: Thrombocytopenia; Malaria; Low Platelets

\section{Introduction}

Malaria continues to be a major health problem in more than 100 endemic countries located primarily in tropical and sub-tropical regions around the world [1]. Malaria is a severe disease caused by parasites of the genus Plasmodium, which is transmitted to humans by a bite of an infected female mosquito of the species Anopheles. Malaria remains the leading cause of mortality around the world, and early diagnosis and fast-acting treatment prevent unwanted outcomes [2]. Hematological changes are some of the most common complications in malaria and they play a major role in malaria pathogenesis. Patients infected with malaria exhibited important changes in most of hematological parameters with low platelet, WBCs, and lymphocyte counts being the most important predictors of malaria infection [3]. Alterations of blood coagulation are thought to be involved in malaria pathogenesis. Also, in malaria patients without clinically evident bleeding or thrombotic complications, alterations of the blood coagulation system, such as decreased levels of plasma antithrombin or elevated levels of plasminogen activator inhibitor (PAI)-1, and thrombocytopenia are frequently found $[1,5,6]$.

Most of researchers reported that, Anemia thrombocytopenia are the most frequent malaria-associated hematological complications and have received more attention in the scientific literature due to their associated mortality [7,8]. The anemia is usually due to varied reasons ranging from hemolysis to comorbidities like parasitic infections, folate, iron, and vitamin B12 deficiencies in endemic areas, antimalarials and further complicated by the coexistence of thalassemia and other haemoglobinopathies $[9,10]$. Regarding to thrombocytopenia during malaria however is yet to be understood clearly and what the mechanism exactly leads to lower the number of thrombocytes during malaria, some researchers hypothesize that malaria parasite produces factors that reduce the platelet production from the megakaryocytes. This saves the parasite from the platelet mediated clearance as a survival mechanism [11]. Other studies reported the mechanisms leading to thrombocytopenia are coagulation disturbances, splenomegaly, bone marrow alterations, antibody-mediated platelet destruction, oxidative stress, and the role of platelets as cofactors in triggering severe malaria $[9,12,13,14,15]$. Fajardo and Tallent demonstrated Plasmodium vivax within platelets by electron microscopy and suggested a direct lytic effect of the parasite on the platelets [16]. Multiple studies indicate that platelets appear to both ameliorate infection by killing parasites and compromise the host by mediating adhesion of infected erythrocytes to the vascular wall. Herein, the possible causes of malaria-induced thrombocytopenia as well as the roles of the platelets in the erythrocytic stage of the disease will be discussed [17]. Another study conducted by Steven Kho et al.,

\footnotetext{
${ }^{*}$ Corresponding author: Asaad Ma. Babker
}

Copyright (C) 2020 Author(s) retain the copyright of this article. This article is published under the terms of the Creative Commons Attribution Liscense 4.0. 
demonstrates the direct pathogen-killing actions and host protective roles of platelets during human malarial infection. Given platelets show broad-spectrum antimicrobial activity, and the risks for infection associated with thrombocytopenia [18]. Another hypothesis released by R. Conte et al., that autoantibodies against platelet glycoproteins IIb-IIIa and Ia-IIa might be present during malaria and could lead to severe thrombocytopenia [19]. Lee SH et al., conducted clinical trial in plasma macrophage colony-stimulating factor and P-selectin levels in malaria and they suggest that elevated M-CSF in malaria, by enhancing macrophage activity, may result in increased macrophagemediated platelet destruction [20]. Another study conducted by Casals et al., in Kenya about thrombocytopenia among patients with falciparum malaria and find thrombocytopenia strongly correlates with high levels of interleukin (IL)-10 [21]. Kotepui et al., observed patients during infected with malaria exhibited important changes in most of hematological parameters with low platelet, WBCs, and lymphocyte counts being the most important predictors of malaria infection. When used in combination with other clinical and microscopy methods, these parameters could improve malaria diagnosis and treatment [22].

\section{Conclusion}

Malaria is associated with different degrees of low platelet count generally mild or moderate and bleeding is rare even during severe malaria. Thrombocytopenia can used as indicator value for presence of malaria after excluding others causes. The relationship between thrombocytopenia and malaria not sufficiently understood till now. Further evaluation of thrombocytopenia should be undertaken during malaria.

\section{References}

[1] Kar NP, Kumar A, Singh OP, Carlton JM and Nanda N. (2014). A review of malaria transmission dynamics in forest ecosystems. Parasites \& vectors, 7, 265.

[2] Talapko J, Škrlec I, Alebić T, Jukić M and Včev A. (2019). Malaria: The Past and the Present. Microorganisms, 7(6), 179.

[3] Kotepui M, Phunphuech B, Phiwklam N, et al. (2014). Effect of malarial infection on haematological parameters in population near Thailand-Myanmar border. Malar J, 13, 218.

[4] Angchaisuksiri P. (2014). Coagulopathy in malaria. Thromb Res, 133, 5-9.

[5] Riedl J, Mordmüller B, Koder S, Pabinger I, Kremsner PG, Hoffman SL, Ramharter M and Ay C. (2016). Alterations of blood coagulation in controlled human malaria infection. Malaria journal, 15, 15.

[6] Asaad Ma. Babker. (2020). The role of Inherited Blood Coagulation Disorders in Recurrent Miscarriage Syndrome. Journal of Critical Reviews, 7 (1), 16-20.

[7] Lacerda MVG, Mourão MPG, Coelho HCC and Santos JB. (2011). Thrombocytopenia in malaria: who cares? Memorias do Instituto Oswaldo Cruz, 106, 52-63.

[8] Wickramasinghe SN and Abdalla SH. (2000). Blood and bone marrow changes in malaria. Baillieres Best Pract Res Clin Haematol, 13, 277-299.

[9] Khan SJ, Abbass Y and Marwat MA. (2012). Thrombocytopenia as an indicator of malaria in adult population. Malaria research and treatment.

[10] K Ghosh. (2007). "Pathogenesis of anemia in malaria: a concise review," Parasitology Research, 101(6), 14631469.

[11] Srivastava K, Sharma M and Mitchell WB. (2017). Malaria and Thrombopoiesis: A Possible Mechanism for the Malarial Thrombocytopenia, 6, 2.

[12] A Rasheed, S Saeed and SA Khan. (2008). "Platelet count in malaria," Pakistan Journal of Phytopathology, 19(3), 86-88.

[13] SS Jamal Khan, FR Khan, M Usman and S Zahid. (2008)."Malaria can lead to thrombocytopenia,” Rawal Medical Journal, 33(2), 183-185.

[14] TS Faseela, RA Roche, KB Anita, CS Malli and Y Rai. (2011). “Diagnostic value of platelet count in malaria,” Journal of Clinical and Diagnostic Research, 5(3), 464-466.

[15] Mustafa ME, Mansoor MM, Mohammed A and Babker AAA. (2015). Evaluation of platelets count and coagulation parameters among patients with liver disease. World Journal of Pharmaceutical Research, 4(10), 360-368. 
[16] Fajardo LF, Tallent C. (1974).Malarial parasites within human platelets. JAMA, 229, 1205-9.

[17] McMorran BJ. (2019). Immune role of platelets in malaria. ISBT Science Series, 14(1), 67-76.

[18] Kho S, Barber BE, Johar E, Andries B, Poespoprodjo JR, Kenangalem E and Woodberry T. (2018). Platelets kill circulating parasites of all major Plasmodium species in human malaria. Blood, The Journal of the American Society of Hematology, 132(12), 1332-1344.

[19] Conte R, Tassi C, Belletti D, Ricci F and Tazzari PL. (2003). Autoimmune thrombocytopenia in malaria. Vox sanguinis, 85(3), 221-221.

[20] Lee SH, Looareesuwan S, Chan J, Wilairatana P, Vanijanonta S. Chong SM and Chong BH. (1997). Plasma macrophage colony-stimulating factor and P-selectin levels in malaria-associated thrombocytopenia. Thrombosis and haemostasis, 77(02), 289-293.

[21] Casals-Pascual C, Kai O, Newton CR, Peshu N and Roberts DJ. (2006). Thrombocytopenia in falciparum malaria is associated with high concentrations of IL-10. The American journal of tropical medicine and hygiene, 75(3), 434436.

[22] Kotepui M, Phunphuech B, Phiwklam N, Chupeerach C and Duangmano S. (2014). Effect of malarial infection on haematological parameters in population near Thailand-Myanmar border. Malaria journal, 13, 218.

\section{How to cite this article}

Asaad Ma. Babker. (2020). Is thrombocytopenia considered a valuable indicator tool for malaria? GSC Advanced Research and Reviews, 2(3), 52-54. 